\title{
Resiliencia familiar, comprensión, campos de aplicación, aportes y desafíos*
}

\section{Family resilience: understanding, fields of application, contributions and challenges}

\author{
Ricardo A. Jaramillo-Moreno** \\ ORCID 0000-0002-7220-6978 \\ Universidad Santo Tomás, Colombia \\ Recibido: 26 de febrero de 2016 \\ Revisado: 10 de marzo de 2017 \\ Aceptado: 23 de abril de 2017
}

\section{Resumen}

Se realizó una revisión de literatura en la que se pretendió establecer el estado de la investigación y conceptualización del fenómeno de la resiliencia familiar y sus aportes entre los años 2010 y 2016. Se encontró que el estado de la investigación y conceptualización, si bien es aún incipiente, se observa una tendencia al aumento de trabajos de tipo empírico y teórico reflexivo, lo que implica una mayor relevancia frente a la investigación e intervención. Con respecto a las áreas de trabajo se observó la predominancia de: salud, neurociencias aplicadas y rehabilitación, social, comunitaria y de las organizaciones, seguidas por psicología clínica y prevención, psicología militar y en último lugar la educación, en su orden. Se concluyó que resulta imperante la continuidad en el desarrollo de estudios al respecto del tema y ampliar áreas de estudio como la psicología ambiental, política, de tránsito, de las organizaciones y el trabajo.

Palabras clave: resiliencia, resiliencia familiar, familia.

* $\quad$ Artículo de Investigación. DOI: http://dx.doi.org/10.15332/s1794-9998.2017.0002.09

** Correspondencia: Ricardo Jaramillo. Grupo de Investigación XXXXXX. Facultad de Psicología. Universidad Santo Tomás. Dirección postal: Campus San Alberto Magno, Autopista Norte calle 205, vía Arrayanes, km 1.6, Bogotá, Colombia. Correo electrónico: ricardojaramillo@usantotomas.edu.co. 


\section{Abstract}

We conducted a literature review to establish the state of the art and conceptualisation of family resilience between 2010 and 2016. Even if still incipient, there is an increase in both empirical and theoretical-reflective research, which involves a higher relevance in terms of research and intervention. Predominant research areas were: health, applied neuroscience and rehabilitation, social, community and organizational; followed by clinical psychology and prevention, military psychology, and education. Further research is definitely needed to continue expanding the literature and including areas such as environmental psychology, politics, mobility, organizations and work is also important.

Keywords: resilience, family resilience, family.

\section{Introducción}

La pertinencia de realizar una revisión de literatura en resiliencia familiar surge por dos aspectos: el primero, resulta parte del proyecto de investigación: Resiliencia en familias del Ejército donde uno de sus miembros se encuentra privado de la libertad, desarrollado por la Facultad de Psicología de la Universidad Santo Tomás de Aquino, Bogotá y la Dirección de Familia del Ejército Nacional.

El segundo concierne precisamente a la necesidad de establecer cuál es el estado del fenómeno y las investigaciones recientes al respecto, en tanto en los últimos 30 años (Pereira, 2007) el estudio de la resiliencia se ha direccionado hacia la resiliencia infantil y la resiliencia individual (Werner y Smith, 1982, Cyrulnik, 2013), y particularmente en situaciones que se pueden enmarcar como traumáticas o donde la resistencia de la persona es puesta a prueba y cuyo trasfondo representa crisis, ansiedad o sufrimiento.

La resiliencia se considera como la capacidad de un individuo de enfrentar situaciones potencialmente traumáticas y recuperarse, salir fortalecido y con más recursos (Werner y Smith, 1982), su énfasis se orienta hacia el trauma derivado del impacto de situaciones de diverso orden como: crisis sociales, tragedias naturales o resultado de eventos sociales, la emergencia de la adversidad, la vulnerabilidad y el estrés; y más aún, que ese impacto tiene su particular génesis en la infancia, aunque también se puede presentar en toda la trayectoria de vida, pero que esencialmente es trascendido por el individuo en determinadas condiciones que facilitan la capacidad de protegerse de tales situaciones y el desarrollo de una conducta de vida positiva (Pereira, 2007).

Ahora, si bien Werner y Smith (1982), Cyrulnik, (2013), Rutter (2006) plantean que una de las piedras angulares de esta capacidad se centra en la existencia de relaciones de apoyo, enriquecimiento vital, cariño o amor en que las personas, o históricamente los niños, tienen un espacio de transformación o formación que permite dotar de nuevos significados los traumas y superarlos; pareciera que la resiliencia es resultado únicamente de la presencia de estas variables, o por otro lado que "la resiliencia se forja a causa de la adversidad, $o$ las dificultades a las que nos enfrentamos en la vida ayudan a que aparezca lo mejor que hay en nosotros" (Pereira, 2007, p.4) (Las cursivas son nuestras).

De esta manera, y en busca de ampliar la comprensión y sustrato del concepto, a partir de la inclusión de la ecología humana (Bronfenbrenner, 1987), la relación sistémica yo-tú-nosotros-mundo o sistémica (Bertalanffy, 1968), los sistemas amplios (ImerBlack, 1988), compleja en tanto principios como retroactividad, auto-eco-organización, dialógico, hologramático, y auto organización (Morín, 2007) y construccionista social (Berger y Luckmann, 2003; Luhman, 1998; Epson y White, 1993).

Lo anterior, en tanto la persona como ser-en-elmundo, si bien posee una vida de gran riqueza individual, y es fuertemente influenciada y constituida por el mundo, es también ella dentro del marco de las relaciones eco-eto-antropológicas, la constructora y transformadora del mundo, pues lo dota de significado y además, opera sobre él. 
Desde esta lógica y a partir de la inclusión de la familia como unidad eco-sistémica de sobrevivencia (Hernández, 2008) es que, no solo tiene sentido, sino que demandaría profundizar desde lo anterior en la resiliencia y más en la resiliencia comunitaria, social, educativa, organizacional y desde nuestro propósito la familiar.

En cuanto a la resiliencia familiar, esta "designa los procesos de superación y adaptación que tienen lugar en la familia como unidad funcional" (Hernández, 2008, p.40), además de que "procuraría identificar y destacar ciertos procesos interacciónales esenciales que permiten a las familias soportar y salir airosas de los desafíos disociadores" (Walsh, 2005, p.25).

De esta manera, el énfasis sobre la familia como socializadora y sistema constructor de resiliencia, también implicaría observar aspectos que le son propios como los rasgos éticos y morales inmersos en la cultura, el clima familiar, ciclo vital, la socialización basada en el género, la identidad y procesos familiares.

De esta manera y haciendo énfasis en la importancia de ver la resiliencia en el marco de la familia, y desde una posición construccionista social, sistémica, ecológica y compleja, este artículo se plantea como interrogante: ¿cuál es el estado del desarrollo teórico e investigativo de la resiliencia familiar en la actualidad?

Para responder a lo anterior, se realizó una revisión de literatura que inicialmente consideró una muestra de 61 artículos pero que se depuró teniendo en cuenta una ventana de observación comprendida entre 2010 y 2016, se tuvo en cuenta artículos en inglés, español y portugués, y se utilizaron palabras clave como resiliencia familiar o family resilience; en cuanto a métodos de investigación fueron incluidos aquellos reportados como cuantitativos, cualitativos o mixtos; Se utilizaron como fuentes de consulta: google académico, scielo, academic search, J stor, doaj y ebsco host; Posteriormente los artículos seleccionados se redujeron a 53 y los 8 restantes que fueron publicados entre 2005 y 2009 se tomaron como fuentes teóricas adicionales.
Para realizar el estudio de resultados se hizo un ejercicio de análisis categorial en el que se destacaron los campos de trabajo en resiliencia familiar, vale aclarar que si bien este ejercicio se refirió a la campos disciplinares psicológicos, un resultado evidenciado es que el trabajo en resiliencia es de orden transdisciplinar, en tanto ciencias como la enfermería (Borges y Silva, 2010), medicina, trabajo social (Distelberg \&Taylor, 2013; Greif, 2012), y economía (Ungar, 2010), presentan interesantes hallazgos.

De esta manera, se plantearon categorías de análisis como: psicología clínica y prevención, salud, neurociencias aplicadas y rehabilitación social, comunitaria y de las organizaciones, educación, psicología militar, conceptualización de la resiliencia y la familia.

De esta forma y en cuanto a la ventana de observación se puede ver que con respecto al año de publicación, en 2010 y 2011 se presentó un mayor número de publicaciones con un $54 \%$ y el $46 \%$ restante entre el 2012 y 2016.

Tabla 1.

Distribución de número de publicaciones entre 2010 y 2016

\begin{tabular}{ccc}
\hline Año & Número de artículos & $\%$ \\
\hline 2010 & 15 & 28,3 \\
\hline 2011 & 14 & 26,4 \\
\hline 2012 & 8 & 15,1 \\
\hline 2013 & 9 & 17 \\
\hline 2014 & 2 & 3,77 \\
\hline 2015 & 4 & 7,55 \\
\hline 2016 & 1 & 1,89 \\
\hline
\end{tabular}

De acuerdo al tipo de publicaciones se encontró que aproximadamente el $80 \%$ fueron artículos empíricos y el $20 \%$ de revisión o reflexión, lo que de alguna manera sugiere la importancia que ha tomado la investigación aplicada en la resiliencia familiar, lo que representa un interés adicional de continuar desarrollando ejercicios aplicados, o investigaciones empíricas como sistematización de experiencias, investigaciones de corte experimental y estudios de tipo interpretativo o comunitario. 
En cuanto a los campos de trabajo se encontró que las áreas de mayor publicación fueron salud, neurociencias aplicadas y rehabilitación social, comunitaria y de las organizaciones, seguidas por psicología clínica y prevención, psicología militar y en último lugar la educación.

Tabla 2.

Distribución de publicaciones por campos disciplinares publicados entre 2010 y 2016.

\begin{tabular}{lcc}
\hline Campos disciplinares & Artículos & $\%$ \\
\hline Psicología clínica y prevención & 9 & 16,98 \\
\hline $\begin{array}{l}\text { Salud, neurociencias aplicadas } \\
\text { y rehabilitación }\end{array}$ & 14 & 26,42 \\
\hline $\begin{array}{l}\text { Social, comunitario } \\
\text { y de las organizaciones }\end{array}$ & 14 & 26,42 \\
\hline Educación & 1 & 1,887 \\
\hline Psicología militar & 9 & 16,98 \\
\hline $\begin{array}{l}\text { Conceptualización } \\
\text { de la resiliencia y la familia }\end{array}$ & 6 & 11,32 \\
\hline
\end{tabular}

Ahora, al realizar un análisis por cada una de las categorías se puede comentar que al respecto del campo de salud, las neurociencias y rehabilitación se encontraron estudios generales que exploran la capacidad de resiliencia de la familia o lo que se puede denominar competencias familiares resilientes frente a dos fenómenos: el trabajo en ambiente clínico, en tanto, no solo se habla de la salud misma, sino de cómo la relación paciente-personal de la salud, resulta un factor primordial en la salud, el desarrollo y la calidad de vida, lo que representa en síntesis, el valorar los cuidados hospitalarios y atención primaria, tal como lo plantean Borges y Silva (2010) al trascender la relación médicopaciente a médico-paciente-familia.

El otro foco de interés se orienta hacia la psicología de la salud, y lo que se puede concebir como evento crítico, que en este caso es la presencia de enfermedades congénitas, crónicas, por virus, bacterias, accidentes, entre otros; en tanto estas no solo representan malestar o deterioro al paciente, pues en todos los casos enferma toda la familia, en tanto su dinámica, estructura e identidad.

Ante estas situaciones, el trabajo con resiliencia familiar resulta de gran ayuda tal como lo señalan Hamall, Heard, Inder, McGill y Kay-Lambkin (2014), o de forma particular los estudios aplicados a la cardiopatía congénita (Jackson, Frydenberg, Liang, Higgins \& Murphy, 2015), el afrontamiento en la enfermedad mental de los padres (Foster, O'Brien \& Korhonen, 2012) trastornos mentales serios (Zauszniewski, Bekhet \& Suresky 2010), cáncer (Yang, Moo-Hee \& Yoon, Gyung-Ja, 2012), lesiones traumáticas a nivel de medula espinal (Simpson y Jones 2012), o incluso la afectación a los cuidadores primarios cuando estos son su familia, en los casos de discapacidad en tercera edad (Cárdenas y López ,2010), niños con pérdida auditiva (Ahlert \& Greeff , 2012), desordenes con espectro autista (Bekhet, Johnson \& Zauszniewski, 2012), VIH y consumo de SPA (Beard, Biemba, Brooks, Costello, Ommerborn, Bresnahan, Flynn, Simon, 2010), Manejo del dolor crónico (West, Usher, Foster, 2011) autismo infantil (Greeff \& Van der Walt, 2010), y discapacidad visual (Klerk, \& Greeff, 2011), lo anterior sugiere que no solo en el afrontamiento del proceso de enfermar es importante para el trabajo con el paciente, sino para su pronóstico y evolución, pues al incluir la familia y sus competencias estas resultarían de enorme valor, particularmente como lo sugeriría Pereira (2007) respecto al enfrentamiento de la situación y la conducta vital positiva.

Por otro lado, y retomando los inicios del trabajo en resiliencia humana, el área que mayor desarrollo refiere es la social, comunitaria y de las organizaciones, así mismo es importante comentar, que si bien comprende la psicología social y comunitaria, es un campo que posee nexos con el concepto de salud mental, desarrollo social y vulnerabilidad, al punto que en algunos casos hay aportes de la economía, y su relación con fenómenos como: violencia política, de masas, intrafamiliar y a la niñez, el desplazamiento y la inmigración.

De tal manera y siguiendo los primeros experimentos de Werner y Smith (1982) frente a la infancia y la marginalidad, se observa la importancia del trabajo con redes de apoyo y resiliencia comunitaria (Juliano \& Yunes, 2014), modelos socioeconómicos, y recursos sociales para la salud mental (Ungar, 2010), apoyo social en casas comunitarias (Distelberg \& Taylor, 2013), salud comunitaria y marginación (Landau, 2010) 
y el desarrollo económico y empresas familiares (Acquaaha, Amoako-Gyampahb \& Jayaram, 2010), bajos ingresos familiares y funcionamiento familiar (Bhana, \& Bachoo, 2011) o el desarrollo de alternativas para mejorar la calidad de vida a partir de la recursividad como las empresas familiares rurales y urbanas (Brewton, Danes, Stafford, Haynes, 2010), lo anterior significaría que aspectos de orden social, político y económico, pueden introducir de alguna manera deterioro o enfermedad en la relación ecosistémica familia-comunidad-país-sistemas amplios, y que una alternativa que permita la inclusión, la justicia, trasformación y por consecuencia la salud, sería el trabajo sobre la familia y la puesta en marcha de competencias que se sirvan del ingenio, la perseverancia, la proactividad y el intercambio de recursos entre ellas y la comunidad.

En complemento y como resultado de: crisis económicas, sociales, humanitarias y el fenómeno de la explosión demográfica emergen fenómenos como la inmigración y el desplazamiento, que per se representan dificultades de adaptación en los sistemas familiares y que consecuentemente derivan o pueden derivar en enfermedades mentales como la depresión, y donde la resiliencia familiar es de gran interés como es el caso de la inmigración en el contexto oriental (Yu, Stewart, Liu,\& Lam, 2013) en la medida que se activan mecanismos de apoyo familiar a nivel nuclear, extenso, al punto de trascender hacia la comunidad, y derivando en la aceptación de situación adversa, la cohesión, el apoyo colectivo y la protección.

No obstante y considerando la emergencia de conflictos políticos, raciales e incluso religiosos en el mundo y su derivación en la guerra, terrorismo o intervenciones armadas, particularmente, como el de la Franja de Gaza y el conflicto palestino israelí, y donde el trabajo en la familia representa un gran valor (Finklestein, 2016), o en el caso donde se da el desplazamiento forzado y se requiere el trabajo con refugiados y reasentamiento familiar (Levin, Hakizimana, Dahnweih, 2011).

La última área de lo social se refiere a la recuperación de las familias que han tenido pautas relacionales violentas o de riesgo de violencia intrafamiliar y que reportan casos exitosos de reunificación en situaciones de custodia removida por maltrato infantil (Greif, 2012) o la recuperación frente a la exposición infantil a violencia intrafamiliar (Kathryn \& Howell, 2011).

En cuanto a la psicología clínica y prevención se observó un especial énfasis en intervenciones familiares de orden sistémico que asisten a consulta y que refieren situaciones de riesgo, donde el entrenamiento en competencias familiares ha sido de gran valor (Seidl, Lurdes, Benetti, y Pereira, 2011), así como el afrontamiento del estrés, recuperación post crisis y ajuste familiar (McCubbin \& McCubbin, 2013), las competencias familiares y prevención de riesgos (Borden, Schultz, Herman \& Brooks, 2010) y el ocio y funcionamiento familiar (Hornberger, Zabriskieb \& Freeman, 2010).

También se observó el trabajo preventivo frente a la crianza en madres jóvenes y riesgo de maltrato infantil (Easterbrooks, Chaudhuri, Bartlett \& Copeman, 2011), el desarrollo positivo ante el estrés en contextos culturales (Ungar, 2010), la salud mental y el VIH (Betancourt, Meyers-Ohki, Stulac, Barrera, Mushashi, Beardslee, 2011), el duelo ante la pérdida de un hijo (Greeff, Vansteenwegen \& Herbiest, 2011), las situaciones de alto riesgo (Lietz, 2012), y el duelo en el marco de la comunidad y sentido de vida (Hooghe \& Neimeyer, 2012).

Así, si bien la psicología clínica se vincula con el concepto de salud, salud mental y su contraparte la enfermedad mental, la orientación en psicología clínica es claramente preventiva y positiva, u orientada al trabajo con problemas derivados de la ansiedad, el estrés y en menor medida con el duelo; por último y en el orden de la salud mental dentro de la escuela, se observó la experiencia exitosa frente a la forma que se enfrenta el bullying con el apoyo familiar en niños y la resiliencia (Bowes, Maughan, Caspi, Moffitt, Arseneault, 2010).

Retomando uno de los argumentos que justifican esta revisión, la resiliencia familiar en el Ejército o las Fuerzas Militares en su conjunto, en tanto se incluyen la Armada, la Fuerza Aérea y Marines (Infantería de Marina), y su misionalidad, frente a los órdenes constitucionales de diversos países vinculados a la Organización del Tratado de 
Atlántico Norte (OTAN) y el requerimiento de velar y ofrecer bienestar a sus miembros y familias conlleva varias consecuencias relacionadas con dificultades de adaptación, estructura y funcionalidad familiar, lo que se encuentra conectado a la afectación en la salud, salud familiar, salud mental y calidad de vida.

Muestra de esto son los efectos en la estructura y dinámica familiar como resultado del despliegue de sus miembros en situaciones del servicio o en medio del combate, el cual no se da necesariamente en situación de guerra regular (Chapin, 2011; Lester, Leskin, Woodward, Saltzman, Nash, Mogil, Paley, Beardslee, 2011; Paley, Lester \& Mogil, 2013), lucha contra el terrorismo (MacDermid, 2010), o cuando dentro de las anteriores situaciones se da la separación por despliegue, como el caso de las tropas norteamericanas que se movilizaron a Afganistán, Siria o Irak, y puntualmente se observa la relación con problemas que requieren atención en salud mental y la disfunción familiar (Riggs \& Riggs, 2011), pero que desde las intervenciones basadas en resiliencia familiar se observa el ostensible desarrollo de fortalezas en la familia, manejo y control de las situaciones de estrés y el crecimiento familiar positivo, además del ajuste psicológico a lo largo de las etapas de despliegue.

Con la presencia de trastornos mentales derivados de la guerra, se observa el beneficio en cuanto al afrontamiento familiar y el desarrollo de habilidades para el funcionamiento del matrimonio (Gottman, Gottman \& Atkins, 2011), la salud mental, familiar y la relación ecológica con otros sistemas familiares y comunitarios (Blair, Lester \& Mogil, 2013).

Esto demuestra, que no solo se ha despertado el interés investigativo-interventivo en esta área por sus beneficios, también se da por la responsabilidad ética y moral de los Estados y sus instituciones, para con sus funcionarios y más con las Fuerzas Militares, cuya labor no solo es diferente a otros trabajos, sino de un enorme riesgo físico, mental, relacional y social, sumado a la misión de salvaguardar los países de amenazas extranjeras o internas, en las que incluso se pone en riesgo la propia vida.
Para finalizar, otro de los resultados de la revisión emerge del desarrollo y profundización del fenómeno, además de adherir comprensiones emergentes al respecto de la familia y que pretenden aportar a su aplicación en diversos campos como los previamente enunciados, muestra de lo anterior se observa en una tendencia a trabajar en el desarrollo teórico y empírico de la resiliencia familiar hacia la comprensión ecológica y construccionista social, y en torno a la visión de la estructura e identidades familiares, el afrontamiento de las crisis, vulnerabilidad y desarrollo (Walsh \& Mc Goldrick, 2013).

Así mismo se encuentran desarrollos frente a la inclusión del ciclo vital, en tanto se presta para la transferencia de competencias familiares resilientes (Johnston, Bailey \& Wilson, 2014) y algunos aportes de interés como el estudio de la transferencia genética de predictores de la resiliencia (Bradley, Davis, Wingo, Mercer \& Ressler, 2013), red de apoyo e infancia (Walsh, 2011) y prevención comunitaria (Walsh, 2012).

\section{Conclusiones}

Para concluir, si bien el trabajo en resiliencia humana es relativamente novedoso, su desarrollo actual es limitado, particularmente en el énfasis en la familia; así mismo es importante reconocer la necesidad de incluir la enseñanza y el estudio del fenómeno dentro de los planes de formación de las disciplinas que se orientan hacia la comprensión del ser humano, la salud y el desarrollo social, y enfáticamente desde una visión más positiva de la persona y particularmente en el marco de la familia y la comunidad.

Lo anterior no solamente en la formación, también en la investigación, diseño e implementación de programas que opten por el reenfoque de las diversas intervenciones, pasando de la salud y salud mental, a la salud y salud mental positivas (Jaramillo-Moreno, 2014), el desarrollo a escala humana basado en la potencialidad y la recursividad, de tal manera, que la unidad familia-comunidadsistemas amplios puedan hacer frente a las situaciones de vulnerabilidad, adversidad o crisis, tan apremiantes hoy en día. 
En cuanto a la resignificación de las comprensiones que sustentan epistemológica, ontológica y conceptualmente la familia, es notable ver como el dedicar ejercicios descriptivos, exploratorios, revisiones de literatura o documentos en torno a esta reflexión, sugiere que las intervenciones deben orientarse hacia la inclusión del apoyo, la cohesión, cuidado y el crecimiento, desde posiciones cada vez más orientadas a lo ecológico, sistémico y a creer fundamentalmente en la capacidad de resistencia, entereza y generatividad de las familias, y como estas características redundan en los individuos y su contextos de desenvolvimiento.

En este orden de ideas, es de resaltar la conceptualización de fenómenos como el trauma, la crisis o el estrés, en tanto requieren, como sostiene Michaud (1999), una reconceptualización que esquemáticamente nos invita a considerar que a partir de la experiencia de la familia de hechos o experiencias limite (Jaspers, 1959) o que representan en sí, altísimo estrés o agresión en niveles como el biológico, familiar (la historia y ciclo vital), micro y macro social y de factores como la cantidad, cualidad y previsibilidad e imprevisibilidad de agresión en la familia (Tomliewicz, 2004).

De esta manera, el espectro actual de situaciones que ponen a prueba la capacidad de resistencia, recuperación, entereza y construcción son amplias; y todos se pueden observar en el marco de y a partir de la familia.

Si bien, unos parten de aspectos fuera de su control u otros característicos de la vida cotidiana (vejez, embarazos, abortos, separaciones, cambios laborales, accidentes) pueden contener en sí diversos grados de agresión, a partir de la cual la familia se pone a prueba; también se encuentran los derivados de la vulnerabilidad social (Pizarro, 2001), cuyo resultado deriva en inadaptaciones de los individuos, familias, y la consecuente sobreexposición a situaciones de riesgo en la familia, infancia y adolescencia (Rojas, 1998), violencia o malestar.

En otro nivel, se podría encontrar la experiencia de situaciones que representan la más clara adversidad o tragedia, y que ya retoma Frankl (1994) y que denomina la triada trágica, pues están atravesados por la muerte, la culpa y el sufrimiento o en complemento con May (2000) el aislamiento; y por último las crisis sociales que derivan en guerra, violencia, terrorismo, crisis económicas, marginalidad y pobreza. Todo lo anterior justificaría precisamente la diversidad de aplicaciones del concepto de resiliencia familiar, no solo a nivel de la psicología sino de otras ciencias, como las de la salud, humanas y sociales.

Ahora bien, teniendo en cuenta los campos potenciales de desarrollo, la perspectiva ecológica y específicamente los sistemas amplios, la agresión también puede provenir de la vivencia de situaciones dentro de las organizaciones humanas, y que se relacionan con la calidad de vida y salud mental de la familia, dentro de estas se pueden enunciar particularmente, las vinculadas al campo educativo y ocupacional, lo cual nos invita a pensar en no solo la resiliencia comunitaria (Melillo y Suárez, 2008) sino de las organizaciones y del trabajo, la relación con el medio ambiente, con el tránsito y la vida en la ciudad, y la influencia del crecimiento y desarrollo acelerado en el campo frente a fenómenos como la exclusión o vulneración de los derechos de las minorías, en la medida que tal agresión no solo lleva a la emergencia de fenómenos como el acoso y violencia de masas, sino a la necesidad de crear ejercicios familiares para sobreponerse, resistir y transformar las realidades y sistemas humanos.

\section{Referencias}

Acquaah, M., Amoako-Gyampah, K. \& Jayaram, J. (2011). Resilience in family and nonfamily firms: an examination of the relationships between manufacturing strategy, competitive strategy and firm performance. International journal of production research, 49(18), 5527-5544. Recuperado de http://www.tandfonline.com/ doi/abs/10.1080/00207543.2011.563834

Ahlert, I. \& Greeff, A. (2012). Resilience factors associated with adaptation in families with deaf or hard of hearing children. American Annals of the Deaf, 157(4), 391-404. Recuperado de: http://eric.ed.gov/?id=EJ1001003 
Beard, J., Biemba, G., Brooks, M.I., Costello, J., Ommerborn, M., Bresnahan, M., Flynn, D. \& Simon, J.L. (2010). Children of female sex workers and drug users: a review of vulnerability, resilience and family-centred models of care. Journal of the International AIDS Society, 13(2), 1. doi: 10.1186/1758-2652-13-S2-S6

Bekhet, A.K., Johnson, N.L. \& Zauszniewski, J.A. (2012). Resilience in family members of persons with autism spectrum disorder: A review of the literature. Issues in mental health nursing, 33(10), 650-656. Recuperado de http:// www.tandfonline.com/doi/abs/10.3109/01612 840.2012.671441

Bertalanffy, L. (1968). General system theory: foundations, development, applications. Edmonton, Canadá: University of Alberta.

Betancourt, T.S., Meyers-Ohki, S., Stulac, S.N., Barrera, A.E., Mushashi, C. \& Beardslee, W.R. (2011). Nothing can defeat combined hands (Abashize hamwe ntakibananira): Protective processes and resilience in Rwandan children and families affected by HIV/AIDS. Social science \& medicine, 73(5), 693-701. Recuperado de http://www.sciencedirect.com/science/ article/pii/S0277953611004217

Berger, P. \& Luckman, T. (2003), La construcción social de la Realidad. Buenos Aires, Argentina: Amorrortu.

Bhana, A. \& Bachoo, S. (2011). The determinants of family resilence among families in low- and middle-income contexts: A systematic literature review. South African Journal of Psychology, 41(2), 131-139. doi: 10.1177/008124631104100202

Borden, L., Schultz, T., Herman, K. \& Brooks, C. (Sep. 2010). The incredible years parent training program: promoting resilience through evidence-based prevention groups. Group Dynamics: Theory, Research, and Practice, 14(3), 230-241. http://psycnet.apa.org/index. cfm?fa=buy.optionToBuy\&id=2010-18447-004

Borges, Z. \& Silva M. (2010). Promoción de la esperanza y resiliencia familiar: prácticas apreciativas. Investigación y Educación en Enfermería, 28(2), 250-257. http://www. scielo.org.co/scielo.php?script=sci_ arttext\&pid=S0120-53072010000200012\&lng= en\&tlng=es.

Bowes, L., Maughan, B., Caspi, A., Moffitt, T. E. \& Arseneault, L. (2010). Families promote emotional and behavioural resilience to bullying: Evidence of an environmental effect. Journal of Child Psychology and Psychiatry, 51(7), 809817. doi: 10.1111/j.1469-7610.2010.02216.x

Bradley, B., Davis, T.A., Wingo, A.P., Mercer, K.B. \& Ressler, K.J. (2013). Family environment and adult resilience: contributions of positive parenting and the oxytocin receptor gene. European journal of psychotraumatology, 4, pp. doi: 10.3402/ejpt.v4i0.21659

Brewton, K., Danes, S., Stafford, K. \& Haynes, G. (2010). Determinants of rural and urban family firm resilience. Journal of Family Business Strategy, 1(3), 155-166.

Bronfenbrenner, U. (1987). La ecología del desarrollo humano. Paidós, Ibérica.

Cyrulnik, B. (2013). Los patitos feos. La resiliencia, una infancia infeliz no determina la vida. Bogota: Clave.

Cárdenas, A. \& López, L. (2010). Analysis matrix of resilience in the face of disability, old age and poverty. International Journal of Disability, Development and Education, 57(2). Recuperado de http://www.tandfonline.com/ doi/full/10.1080/10349121003750760

Carvalho, M. \& Mattar, M. (2014). Reflexões sobre rede de apoio social como mecanismo de proteção e promoção de resiliência. Ambiente \& Sociedade, 17(3), 135-154. https://dx.doi. org/10.1590/S1414-753X2014000300009

Chapin, M. (2011). Family resilience and the fortunes of war. Social work in health care, 50(7), 527-542.doi:http://dx.doi.org/10.1080/009813 89.2011 .588130 
DeHann, L., Hawley, D. \& Deal, J. (2012). Operationalizing family resilience as process: proposed methodological strategies. En: Handbook of Family Resilience (pp 17-29). Recuperado de http://link.springer.com/chapter/10.1007\% 2F978-1-4614-3917-2_2

Distelberg, B. \& Taylor, S. (2015). The roles of social support and family resilience in accessing healthcare and employment resources among families living in traditional public housing communities. Child \& Family Social Work, 20(4), 494-506. Recuperado de http://onlinelibrary. wiley.com/doi/10.1111/cfs.12098/abstract

Easterbrooks, M.A., Chaudhuri, J.H., Bartlett, J.D. \& Copeman, A. (2011). Resilience in parenting among young mothers: Family and ecological risks and opportunities. Children and Youth Services Review, 33(1), 42-50. Recuperado de http://www.sciencedirect.com/science/ article/pii/S0190740910002501

Epson, D. \& White, M. (1993). Medios narrativos para fines terapéuticos. Buenos Aires, Argentina: Paidós.

Finklestein, M. (2016). Risk and resilience factors in families under ongoing terror along the life cycle. Contemporary Family Therapy, 38(2), 129- 139. doi:10.1007/s10591-015-9356-4

Foster, K. O’Brien, L. \& Korhonen, T. (2011). Developing resilient children and families when parents have mental illness: A familyfocused approach. International Journal of Mental Health Nursing, 21(1), 3-11. Recuperado de http://onlinelibrary.wiley.com/doi /10.1111/j.1447-0349.2011.00754.x/abstract

Frankl, V. (1994). Logoterapia y análisis existencial. Barcelona, España: Herder.

Gottman, J, Gottman, J. \&, Atkins, C. (2011). The comprehensive soldier fitness program: family skills component. American Psychologist, 66(1), 52-7. doi: 10.1037/a0021706

Greeff, A.P., \& Van der Walt, K.J. (2010). Resilience in families with an autistic child. Education and Training in Autism and Developmental Disabilities, 45(3), 347-355. Recuperado de http://www.jstor.org/stable/23880109

Greeff, A.P., Vansteenwegen, A. \& Herbiest, T. (2011). Indicators of family resilience after the death of a child. OMEGA-Journal of Death and Dying, 63(4), 343-358. doi: 10.2190/OM.63.4.C

Greif, G. (2012). Ambiguous reunification: A way for social workers to conceptualize the return of children after abduction and other separations. Families in Society: The Journal of Contemporary Social Services, 92(2), 305-311. http://www.familiesinsocietyjournal.org/doi/ abs/10.1606/1044-3894.4102?journalCode=fcss

Hamall, K.M., Heard, T.R., Inder, K.J., McGill, K.M. \& Kay-Lambkin, F. (2014). The Child Illness and Resilience Program (CHiRP): a study protocol of a stepped care intervention to improve the resilience and wellbeing of families living with childhood chronic illness. BMC Psychol 2 (5). doi:10.1186/2050-7283-2-5

Hernández, A. (2008). Vínculos, individuación y ecología humana. Hitos para una psicología clínica compleja. Bogotá, Colombia: Editorial de la Universidad Santo Tomás.

Hooghe, A., \& Neimeyer, R.A. (2013). Family resilience in the wake of loss: A meaning-oriented contribution. In Handbook of family resilience (pp. 269-284). Springer New York. Recuperado de http://link.springer.com/chapter/10.1007\% 2F978-1-4614-3917-2_16

Hornberger, L.B., Zabriskie, R.B. \& Freeman, P. (2010). Contributions of family leisure to family functioning among single-parent families. Leisure Sciences, 32(2), 143-161. Recuperado de http://www.tandfonline.com/ doi/abs/10.1080/01490400903547153

Howell, K. (2011). Resilience and psychopathology in children exposed to family violence. Aggression and Violent Behavior 16(6), 562-569. Recuperado de http://www.sciencedirect.com/ science/article/pii/S1359178911000930 
Imber-Black, E. (1988). Familias y sistemas amplios: en terapeuta familiar en el laberinto. Buenos Aires: Amorrortu editores.

Jackson, AC1., Frydenberg, E., Liang, R.P., Higgins, R.O. \& Murphy, B.M. (2015). Familial impact and coping with child heart disease: a systematic review. Pediatr Cardiol, 36(4), 6, [95-712]. doi: 10.1007/s00246-015-1121-9.

Jarrett, T.A. (2013). Warrior Resilience and Thriving (WRT): Rational Emotive Behavior Therapy (REBT) as a resiliency and thriving foundation to prepare warriors and their families for combat deployment and posttraumatic growth in operation Iraqi Freedom, 2005-2009. Journal of Rational-Emotive \& CognitiveBehavior Therapy, 31(2), 93-107. doi:10.1007/ s10942-013-0163-2

Jaramillo-Moreno, R. (2014). Social representations of parents with school-age children concerning risk and protection factors of drug use in Bogota, Colombia. Procedia - Social and Behavioral Sciences, 132(15), 377-383. Recuperado de http://www.sciencedirect.com/ science/article/pii/S1877042814032352

Jaspers, K. (1959). Filosofía. San Juan de Puerto Rico: Universidad de Puerto Rico.

Johnston, J.H., Bailey, W.A. \& Wilson, G. (2014). Mechanisms for Fostering Multigenerational Resilience. Contemp Fam Ther, 36(1), 148-161. doi: 10.1007/s10591-012-9222-6

Landau, J. (2010). Communities that care for families: the LINC model for enhancing individual, family, and community resilience. American Journal of Orthopsychiatry, 80(4), 516-524. Recuperado de http://psycnet.apa. org/journals/ort/80/4/516/

Lester, P., Leskin, G., Woodward, K., Saltzman, W., Nash, W., Mogil, C., Paley, B. \& Beardslee, W. (2010). Wartime deployment and military children: applying prevention science to enhance family resilience. In Risk and resilience in U.S. military families (pp 149-173). New York: Springer. doi: 10.1007/978-1-4419-7064-0_8
Lietz, C.A. (2013). Family resilience in the context of high-risk situations. In Handbook of family resilience (pp. 153-172). New York: Springer. Recuperado de http://link.springer.com/chap ter/10.1007\%2F978-1-4614-3917-2_10

Lietz, C. \& Strength, M. (2011). Stories of successful reunification: A narrative study of family resilience in child welfare. Families in Society: The Journal of Contemporary Social Services, 92(2), 203-210. doi: http://dx.doi. org/10.1606/1044-3894.4102

Luhmann, N. (1990). Sistemas sociales: lineamientos para una teoría general. España: Antropos.

May, R. (2000). Amor y voluntad contra la violencia y la apatia en la sociedad actual. Barcelona, España: Gedisa.

MacDermid, S.M. (2010) Family risk and resilience in the context of war and terrorism. Journal of Marriage and Family, 72, 537-556. doi: 10.1111/j.1741-3737.2010.00717.x

McCubbin, L. \& McCubbin, H. (2013). Resilience in ethnic family systems: A relational theory for research and practice. In Handbook of Family Resilience (pp. 175-195). New York: Springer.

Melillo, A. y Suárez, E. (2008). Resiliencia descubriendo las propias fortalezas. España: Paidos.

Michaud, P.A. (1999). La résilience, un regard neuf sur les soins et la prévention, en Arch. Pédiatriques, 6, 827-831.

Mogil, C., Hajal, N., García, E., Kiff, C., Paley, B., Milburn, N. \& Lester, P. (2015). Focus for early childhood: a virtual home visiting program for military families with young children. Contemp Fam Ther. 37(3): 199-208. doi: 10.1007/s10591015-9327-9. https://www.ncbi.nlm.nih.gov/pmc/ articles/PMC4629808/pdf/nihms667239.pdf

Morín, E. (2007). Introducción al pensamiento complejo. Barcelona, España: Editorial Gedisa.

Paley, B., Lester, P. \& Mogil, C. (2013). Family systems and ecological perspectives on the impact of deployment on military families. Clinical 
Child and Family Psychology Review, 16(3), 245265. doi: 10.1007/s10567-013-0138-y.

Pereira, R. (2007). Resiliencia individual, familiar y social. Interpsiquis. Recuperado de http://www.psiquiatria.com/tratamientos/ resiliencia-individual-familiar-y-social/\#

Pizarro, R. (2001). La vulnerabilidad social y sus desafíos: una mirada desde América Latina. Chile: CEPAL.

Riggs, A. \& Riggs, D. (2011). Risk and resilience in military families experiencing deployment: the role of the family attachment network. Journal of Family Psychology, 25(5), 675-687. doi: 10.1037/a0025286

Rojas, M. (1998). Adolescencia y resiliencia, un comportamiento de riesgo: el consumo de drogas psicoactivas. Psicoactiva 12(6), 39-65.

Rutter M. (2006). Implications of resilience concepts for scientific understanding. Annals New York Academy of Sciences. 1-12.

Saltzman, W., Pynoos, R., Lester, P., Layne, C. \& Beardslee, W. (2013). Enhancing family resilience through family narrative co-construction. Clin Child Fam Psychol Rev. 16(3), 294-310. doi: 10.1007/s10567-013-0142-2

Seidl, A., Lurdes, M., Benetti, D. \& Pereira, S. (2011). Resiliencia familiar: nuevas perspectivas en la promoción y prevención en salud. Diversitas: Perspectivas en Psicología, 7(1), 43-55. Recuperado de http://www.scielo.org.co/ scielo.php?script=sci_arttext\&pid=\$1794-999 $82011000100004 \& \operatorname{lng}=$ en\&tlng=es.

Simpson, G. \& Jones, K. (2012). How important is resilience among family members supporting relatives with traumatic brain injury or spinal cord injury? Clinical Rehabilitation, 27(4), 36777. doi: $10.1177 / 0269215512457961$

Tomkiewicz, S. (2004): El surgimiento del concepto. En B. Cyrulnik y otros, El realismo de la esperanza (pp. 33-50). Barcelona: Gedisa.
Ungar, M. (2010). Families as navigators and negotiators: Facilitating culturally and contextually specific expressions of resilience. Family process, 49(3), 421-435. doi: 10.1111/j.1545-5300.2010.01331.x.

Ungar, M. (2010). What is resilience across cultures and contexts? Advances to the theory of positive development among individuals and families under stress. Journal of Family Psychotherapy, 21(1), 1-16. Recuperado de http://www.tandfonline.com/doi/abs/10.1080/08975351003618494

Wadsworth, S. (2010). Family risk and resilience in the context of war and terrorism. Journal of Marriage and Family. 72(3), 537-556. doi: 10.1111/j.1741-3737.2010.00717.x

Walsh, F. \& Mc Goldrick, M. (2013). Bereavement: A family life cycle perspective. Family Science, 4(1), 20-27. Recuperado de https://www.researchgate.net/publication/296684641_ Family_resilience_a_developmental_systems_ framework

Walsh, F. (2011). Facilitating family resilence: relational resources for positive yputh development in conditions of adversity. The Social Ecology of Resilence, Vol(No.), 173-185. http://link.springer.com/chapter/10.1007\% 2F978-1-4614-0586-3_15

Walsh, F. (2012) Community-Based practice applications of a family resilence framework. In Handbook of Family Resilience (pp. 65-82). New York: Springer. doi: 10.1007/978-1-4614-3917-2_5

Weine, S.M., Levin, E., Hakizimana, L., \& Dahnweih, G. (2011). How prior social ecologies shape family resilience amongst refugees in US resettlement. In The social ecology of resilience (pp. 309-323). New York: Springer. Recuperado de http://link.springer.com/ chapter/10.1007/978-1-4614-0586-3_24.

Werner, E. \& Smith, R. (1982). Vulnerable but invincible: a study of resilient children. New York: McGraw-Hill. 
West, C., Usher, K., \& Foster, K. (2011). Family resilience: Towards a new model of chronic pain management. Collegian, 18(1), 3-10. doi: http:// dx.doi.org/10.1016/j.colegn. 2010.08.004

Yang, Moo-Hee, \& Yoon, Gyung-Ja (2012). A Study on the Development and Effectiveness Verification of a Program Designed to Strengthen Family Resilience of Inpatients with Cancer and Their Families. Journal of the Korean Home Economics Association 50(1), 121-139. doi 10.6115/khea.2012.50.1.121
Yu, X., Stewart, S.M., Liu I.K. \& Lam, T. (2013). Resilience and depressive symptoms in mainland Chinese immigrants to Hong Kong. Soc Psychiatry Psychiatr Epidemiol. 49(2), 241-9. doi: 10.1007/s00127-013-0733-8

Zauszniewski, J, Bekhet, A. \& Suresky, M. (2010) Resilience in family members of persons with serious mental illness. Nursing Clinics of North America 45(4), 613-626. Recuperado de http:// www.sciencedirect.com/science/article/pii/ S0029646510000757 